\title{
Nanostructuring thin polymer films with optical
}

near fields.

Ignacio Martín-Fabiani ${ }^{{ }^{*}}$, Jan Siegel ${ }^{2}$, Stephen Riedel ${ }^{3}$, Johannes Boneberg ${ }^{3}$, Tiberio

A. Ezquerra ${ }^{1}$, Aurora Nogales ${ }^{1}$

${ }^{1}$ Instituto de Estructura de la Materia, IEM-CSIC, Serrano 121, 28006 Madrid, Spain

2 Instituto de Óptica, IO-CSIC, Serrano 121, 28006 Madrid, Spain

${ }^{3}$ University of Konstanz, Fach 676, 78457 Konstanz, Germany

KEYWORDS. Optical near fields, nanostructures, thin films, poly (trimethylene terephthalate), dendritic crystallization, chalcogenides.

ABSTRACT. In the present work, we report on the application of optical near fields to nanostructuring of Poly (trimethylene terephthalate) (PTT) thin films. By exposure to a single ultraviolet nanosecond laser pulse, the spatial intensity modulation of the nearfield distribution created by a silica microsphere is imprinted into the films. Setting different angles of incidence of the laser, elliptical or circular periodic ring patterns can be produced with periods as small as half the laser wavelength used. These highly complex patterns show optical and topographical contrast and can be characterized by Optical Microscopy (OM) and Atomic Force Microscopy (AFM). We demonstrate the key role of the laser wavelength and coherence length in achieving smooth, extended patterns in PTT by using excimer laser $(193 \mathrm{~nm})$ and Nd:YAG laser $(266 \mathrm{~nm})$ pulses. Reference experiments performed in $\mathrm{Ge}_{2} \mathrm{Sb}_{2} \mathrm{Te}_{5}(\mathrm{GST})$ demonstrate that nanopatterning in PTT is triggered by ablation as opposed to GST, in which nanopatterning originates 
from laser-induced phase change, accompanied by a small topographical contrast. The experiments presented in this work demonstrate the suitability of optical near fields for structuring polymer films, opening up new possibilities for nanopatterning and paving the way for potential applications where optical near fields and polymer nanostructures are involved.

\section{INTRODUCTION}

The possibility to overcome the diffraction limit imposed by light wavelength has been a subject of intense study for many years. ${ }^{1}$ The use of Optical Near Fields (ONFs) seems to be one of the best approaches towards overcoming this barrier, resulting in relevant advances in microscopy, ${ }^{2}$ materials processing, ${ }^{3}$ sensors ${ }^{4}$ and phase change memories. ${ }^{5}$ There are mainly two approaches to characterize ONFs, either via direct visualization by techniques such as Scanning Near-Field Optical Microscopy ${ }^{2}$ or by imprinting it on the surface of a certain material. ${ }^{6}$ In fact, one of the most relevant fields in materials processing is laser ablation, ${ }^{7}$ involving different techniques and applications, such as Laser Interference Lithography. ${ }^{8,9}$

Near-field enhancement of incident light can be generated in the presence of metal nanoparticles, ${ }^{10,}{ }^{11}$ dielectric microspheres ${ }^{6}$ or even the tip of an atomic force microscope. ${ }^{12}$ Successful application of ONF generated by dielectric microspheres has been reported in diverse inorganic materials such as silicon, ${ }^{13}$ fused silica, ${ }^{14}$ alumina ${ }^{15}$ and $\mathrm{Ge}_{2} \mathrm{Sb}_{2} \mathrm{Te}_{5}(\mathrm{GST})^{5,}$ 6, 16 by writing sub-micron sized holes at the position of maximum field enhancement. In the case of GST, it has been demonstrated that the imprint is triggered by local laser-induced amorphization leading to optical ${ }^{5,6}$ and morphological ${ }^{16}$ contrast. Topographical contrast in patterns imprinted in GST has been 
observed but only over a very small area with weak modulation amplitude. ${ }^{6}$ Overall, the range of materials that has been proved to be suitable for ONF structuring is narrow and, to the best of our knowledge, does not yet include polymers.

Polymeric materials have become strong candidates in the last years for nanostructuration, ${ }^{17-19}$ since potential applications might benefit from their excellent properties, i.e. mechanical flexibility, light weight, enhanced durability and low cost. Polymer nanostructures are being currently implemented in a wide variety of applications, such as biosensors, ${ }^{20}$ solar cells, ${ }^{17}$ and non-volatile memories. ${ }^{21}$ In particular, Poly (trimethylene terephthalate) (PTT) is a semicrystalline aromatic polyester whose outstanding mechanical and optical properties, together with its low glass transition temperature $\mathrm{T}_{\mathrm{g}} \approx 44^{\circ} \mathrm{C},{ }^{22}$ make it an attractive material for the fiber industry $^{23}$ as well as for optoelectronic ${ }^{24}$ and nanophotonic ${ }^{25,26}$ applications. PTT has already shown interesting features when nanostructured by several different methods such as Laser Induced Periodic Surface Structuration (LIPSS) ${ }^{27}$ and NanoImprint Lithography (NIL). ${ }^{28}$

In the present work we report nanofabrication of two-dimensional near-field topographic patterns imprinted on polymeric thin films of PTT, resulting from interference between incident laser light and light scattered by dielectric microspheres placed at the film surface. The spatial intensity distribution at the film surface plane can be described by the Mie theory ${ }^{29}$ and depends on the properties of the incident light (wavelength, polarization and angle of incidence), the diameter of the sphere and the optical properties of sphere and film. ${ }^{16}$ Different regions can be discerned in the resultant optical field imprinted on the film after irradiation at an angle of incidence $\theta$, as it is shown in Figure 1a. The shadow of the sphere defines a region where the 
material is not affected by the irradiation, except for the small region where the light is focused, leading to strong ablation. The regions outside are exposed to light directly incident from the laser, which interferes with light scattered from the sphere. This leads to interference maxima and minima, with smaller periods in the backward direction than in the forward direction. In the vicinity of the sphere, the extension and shape of the sphere cannot be neglected. In contrast, far from the sphere (Fig. 1b) it can be considered a point scatterer and thus its dimensions can be neglected. In this case, applying the conditions for constructive interference $p_{f w}=\mathrm{b}+\lambda$ and $p_{b w}=\mathrm{a}-\lambda$, expressions can be derived for the period in the forward direction $p_{f w}=\lambda /(1-\sin \theta)$ and in the backward direction $p_{b w}=\lambda /(1+\sin \theta)$.
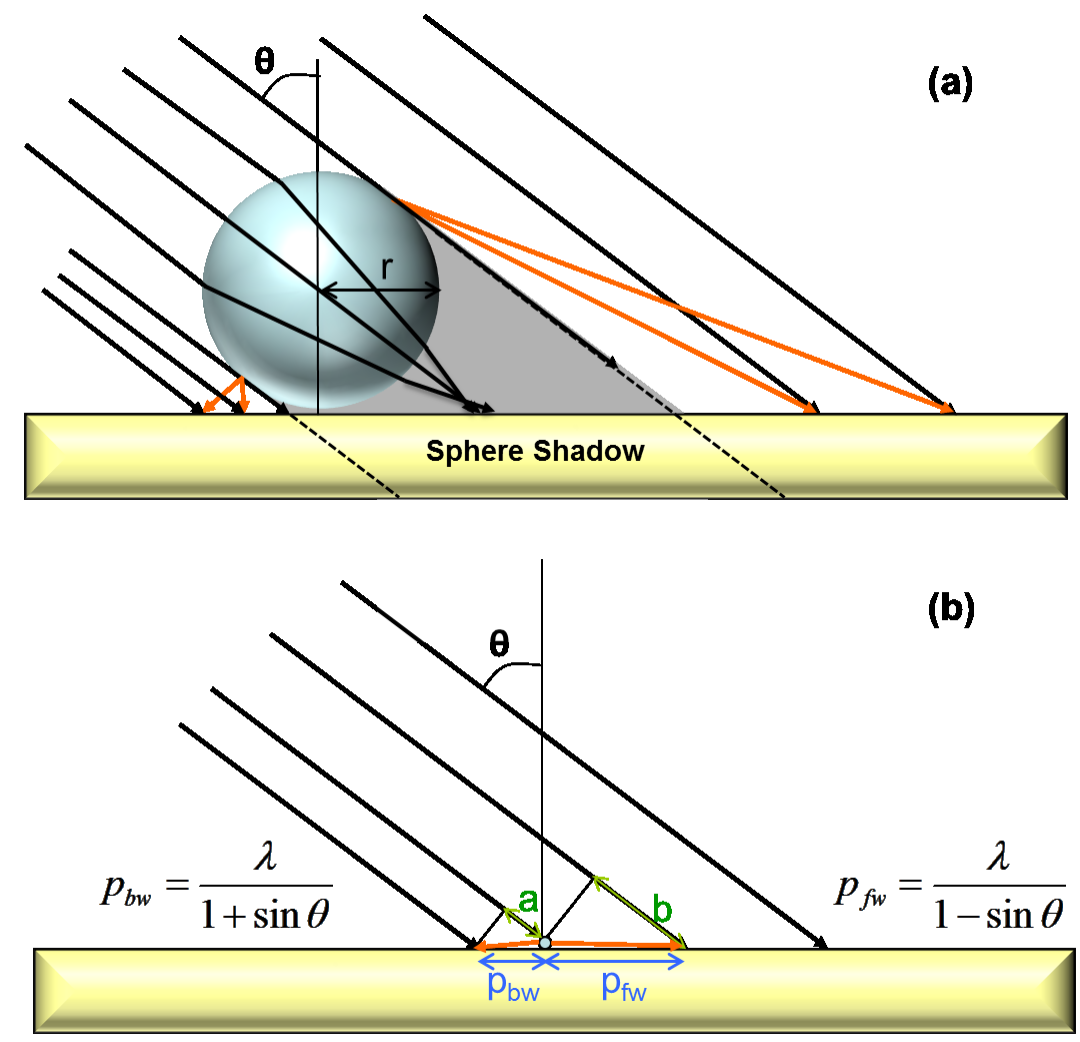

Figure 1. Simplified geometrical construction of the optical field generated by the interference between incident light at an angle $\theta$ and light scattered by a dielectric microsphere of radius $r$ placed at the film surface. The schemes show the construction 
for constructive interference in (a) the vicinity of the sphere and (b) the far field region, including the expressions for the period in the backward $p_{b w}$ and in the forward $p_{f w}$ directions.

Here we demonstrate the concept of near-field patterning applied to polymeric materials, expanding the possibilities of this technique and paving the way for potential applications where optical near-fields and polymer nanostructures are involved. By means of a proper selection of the particle shape, size and properties together with the irradiation parameters (wavelength, pulse duration, angle of incidence) the type of pattern can be designed.

\section{EXPERIMENTAL}

PTT thin films, $150 \mathrm{~nm}$ thick, were prepared by spin-coating of a PTT/trifluoroacetic acid solution $(20 \mathrm{~g} / \mathrm{l})$ onto Si wafers. GST films, fcc crystalline $40 \mathrm{~nm}$ thick, were deposited by sputtering onto $\mathrm{Si}$ wafers with a $10 \mathrm{~nm} \mathrm{SiO} 2$ buffer layer. Silica microspheres, dispersed in isopropanol, with diameter $\Phi=4.6 \mu \mathrm{m}$ were deposited by spin-coating onto the prepared thin films, under conditions that ensure particle isolation of the dielectric microspheres. Samples were irradiated with a single pulse from either an ArF excimer laser (wavelength $\lambda=193 \mathrm{~nm}, 20 \mathrm{~ns}$ pulse duration, top hat intensity distribution with a spot size of $350 \mu \mathrm{m}$ ) or an injection-seeded Nd:YAG laser (wavelength $\lambda=266 \mathrm{~nm}, 10 \mathrm{~ns}$ pulse duration, non-homogeneous intensity distribution with a spot size of $5 \mathrm{~mm}$ ) was applied to isolated silica microspheres at a selected angle $\theta$ (either normal incidence or $\theta=54^{\circ}-60^{\circ}$ ) and a determined fluence $\mathrm{F}$. The absorbance of PTT films was measured using an spectrophotometer UV-Vis Perkin-Elmer U/V Lambda 16, using a quartz sample as a reference for the measurement of the incident 
intensity. PTT films present good absorbance in the UV range, and the linear absorption coefficient values for the irradiation wavelengths are $\alpha_{193}=186490 \mathrm{~cm}^{-1}$ for the excimer laser and $\alpha_{266}=25997 \mathrm{~cm}^{-1}$ for the $\mathrm{Nd}$ : $Y A G$ laser.

After irradiation, patterns were characterized using Optical Microscopy (OM, Nikon Eclipse Ti) and Atomic Force Microscopy (AFM, Nanoscope V, Bruker) in tapping mode. AFM images were analyzed using the software Nanoscope Analysis 1.40. Optical profiles were obtained using ImageJ software, normalizing the reflectivity to 1 in the region where the sample has not been affected by the irradiation. Raman spectra were recorded using a Renishaw Raman InVia Reflex spectrometer, with excitation at $532 \mathrm{~nm}$ and a resolution of $2 \mathrm{~cm}^{-1}$.

\section{RESULTS AND DISCUSSION}

In the first set of experiments the complex optical near and far field intensity distribution generated by a silica microsphere on PTT thin films during excimer laser irradiation has been studied. The laser was incident at an angle of $\theta=54^{\circ}$ and at a fluence $\mathrm{F}=350 \mathrm{~mJ} / \mathrm{cm}^{2}$. The imprint in PTT inspected by optical microscopy (Figure 2) consists of an elliptical periodic ring pattern with a central elliptical region containing a small ablation hole. This hole, approximately in the center of the ellipse, is a crater caused by the focusing effect of the sphere acting as a ball lens, whereas the central elliptical region corresponds to the shadow region of the sphere. Dark rings correspond to regions were the laser fluence has been enhanced locally by the scattered light field of the microsphere. On the other hand, bright rings and the central ellipse correspond to regions exposed to a reduced local fluence with respect to the incident fluence. This ring structure becomes clearer by plotting the cross section of the reflectivity along the 
center of the image (Figure 2, bottom). The silica microsphere is removed during the irradiation process due to the recoil pressure of the ablation.
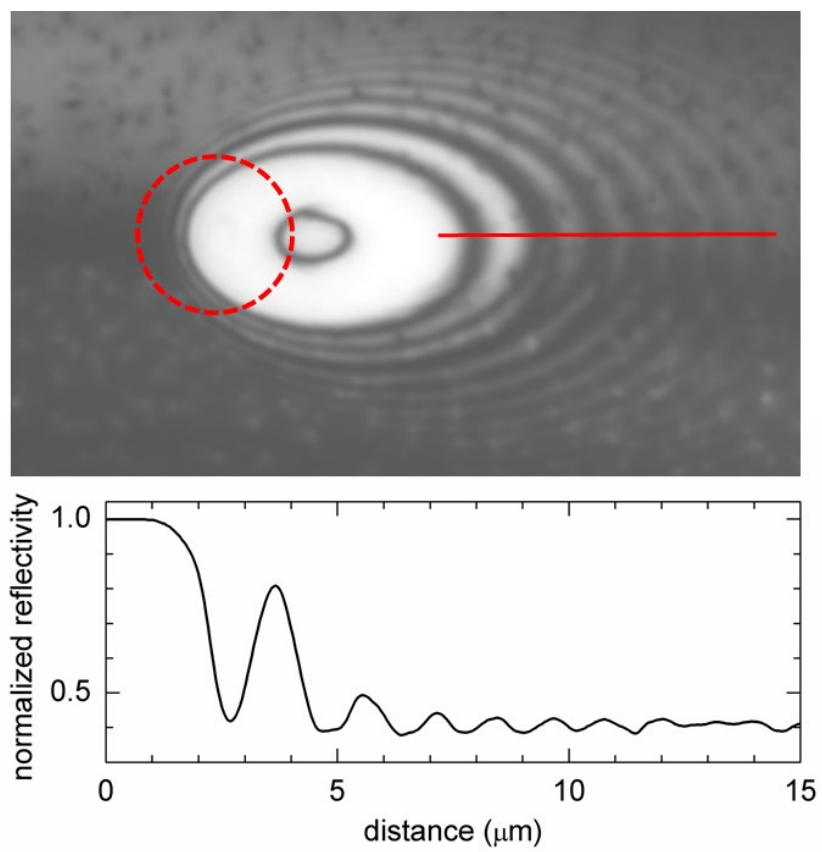

Figure 2. Near-field pattern in PTT film generated by a silica microsphere (diameter $\Phi=4.6 \mu \mathrm{m}$ ) after a single excimer laser pulse irradiation at an angle $\theta=54^{\circ}$ (laser impinges from the left) and fluence $350 \mathrm{~mJ} / \mathrm{cm}^{2}$. Optical micrograph $\left(25 \times 15 \mu \mathrm{m}^{2}\right)$ and reflectivity profile (below) along the red line depicted in the image. The original position of the silica microsphere is marked by a dashed circle.

AFM measurements reveal that the imprinted pattern does not only feature optical but also topographic contrast (Fig. 3). Since the central elliptic region around the main crater corresponds to the shadow region of the microsphere, it serves as a reference for the original thickness of the PTT film $(\mathrm{d}=150 \mathrm{~nm})$. Looking at the height scale it becomes evident that the near-field induced topography modulation extends over the entire film thickness. Moreover, the regions of local fluence enhancement (dark rings in the $\mathrm{OM}$ image) correspond to topographic regions below the initial film surface. Both 
observations point to near-field ablation being the responsible mechanism for patterning in the polymer. ${ }^{30}$

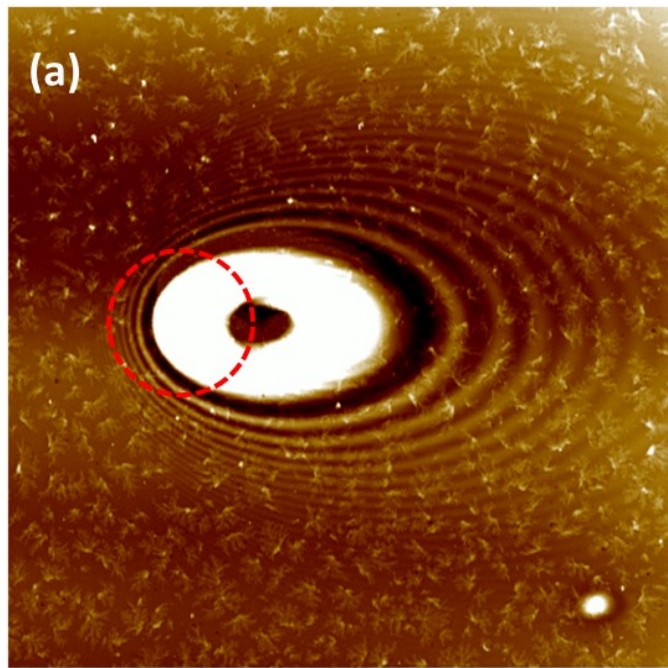

$180 \mathrm{~nm}$
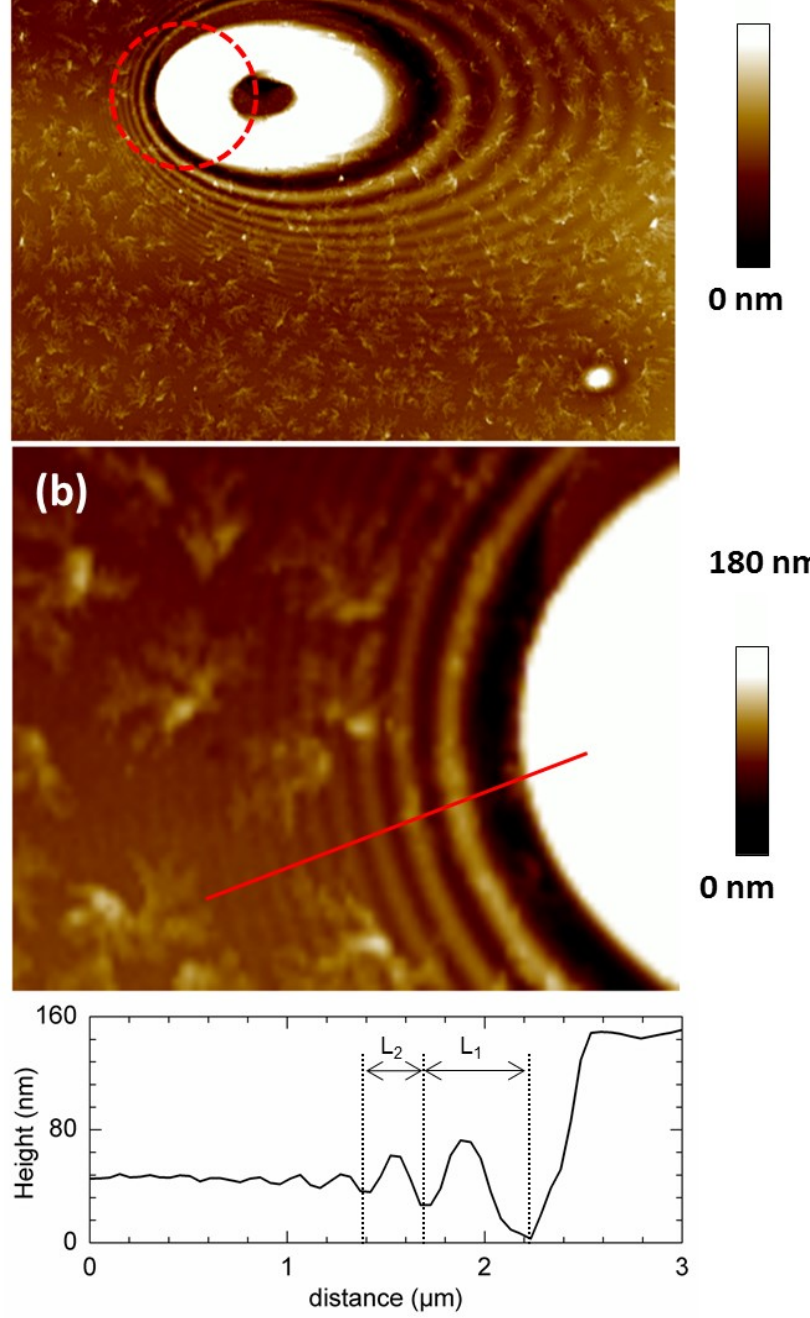

Figure 3. Near-field pattern in PTT film generated by a silica microsphere (diameter $\Phi=4.6 \mu \mathrm{m}$ ) after a single excimer laser pulse irradiation at an angle $\theta=54^{\circ}$ (laser impinges from the left) and fluence $350 \mathrm{~mJ} / \mathrm{cm}^{2}$. (a) AFM topography map $\left(25 \times 25 \mu \mathrm{m}^{2}\right)$ of the same region and (b) zoom $\left(5 \times 4 \mu \mathrm{m}^{2}\right)$ and topography profile (below) along the red line in the backscattering region. The original position of the silica microsphere is marked by a dashed circle. 
One interesting aspect of the irradiated PTT films is the appearance of a characteristic dendritic morphology superimposed to the imprint induced by laser, which are formed within the whole irradiated zone. In a first approach we can attribute this dendritic morphology to polymer crystallization. In order to support this assumption, we performed Grazing Incidence Wide Angle X-Ray Scattering experiments on the BW4 beamline of HASYLAB (DESY, Hamburg, Germany). A full description of the experimental set-up and sample conditions has been previously published. ${ }^{27}$ The experiments we performed on the irradiated and on the non-irradiated sample regions. Fig. 4 shows the diffraction pattern taken with a $0.4^{\circ}$ incidence angle inside for an irradiated zone (red line) and for a non-irradiated one (black line). For the sake of comparison we also added the results for a non-irradiated semicrystalline sample. As one can see, the irradiated sample presents some shoulders for reciprocal lattice q-vector values corresponding to the Bragg peaks of the semicrystalline sample which are not present in the pattern for the non-irradiated sample. Although the effect is weak this results supports that the observed dendrites may be PTT crystals. 


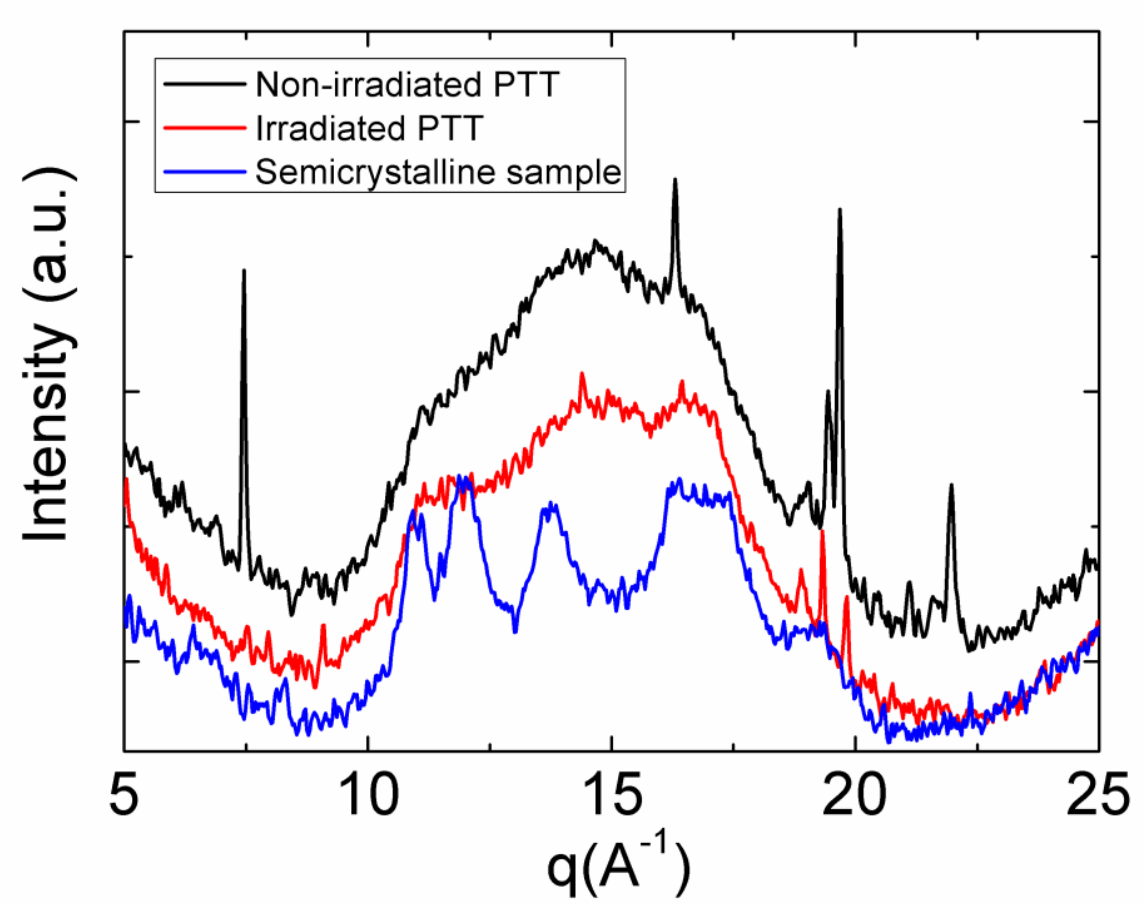

Figure 4. Grazing Incidence Wide Angle X-Ray Scattering (GIWAXS) patterns taken with a $0.4^{\circ}$ incidence angle for (a) non-irradiated zone (red line) and for (b) an irradiated one. For the sake of comparison we also added the results for a non-irradiated semicrystalline sample (c).

In addition, the Raman spectra for the irradiated samples, represented by a red line in Fig. 5, show a strong increase of fluorescence when compared to the spectra of the nonirradiated zone (Fig. 5, black line). This effect can be related to the fact that in PTT, previous to crystallization process, a densification of the material takes place via parallel arrangement of the phenyl rings. ${ }^{31,32}$ This parallel arrangement of the aromatic rings can enhance the fluorescence signal coming from the polymer. This observation further supports a change on the ordering of PTT which in a first approach can be attributed to the dendritic morphology. 


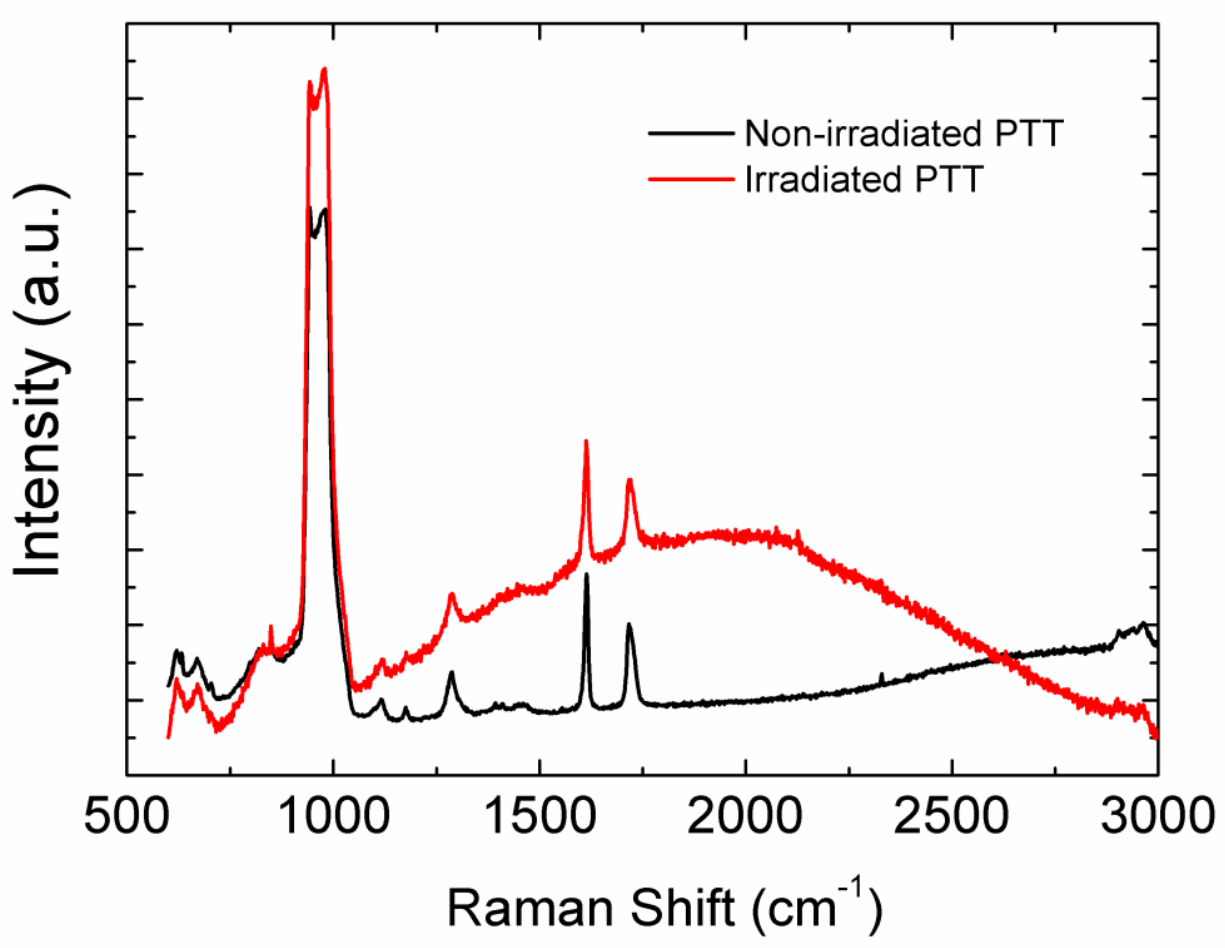

Figure 5. Raman spectra for: (a) the irradiated samples shown by a red line and for (b) non-irradiated ones.

Studying the ONF pattern with AFM in the backward direction (Figure 3b) the resolution of the patterning can be explored, given the improved contrast with respect to the optical images. In the far field, the dielectric microsphere can be considered a single point in space, hence removing any dependence of the fringe period with the sphere properties and allowing to predict the value of the period in the backward direction (see Fig. 1). Considering incidence at $\theta=54^{\circ}$ and the wavelength of the excimer laser (193 $\mathrm{nm})$, the value obtained is $p_{b w, c a l c}=\lambda /(1+\sin \theta)=107 \mathrm{~nm}$. The distance between consecutive height minima as a function of the distance to the position of the first minimum is shown in Figure 6 (filled black circles). Inspecting the graph one can observe that a shortening of the fringe period takes place, becoming closer to the predicted value as the far field is approached. 


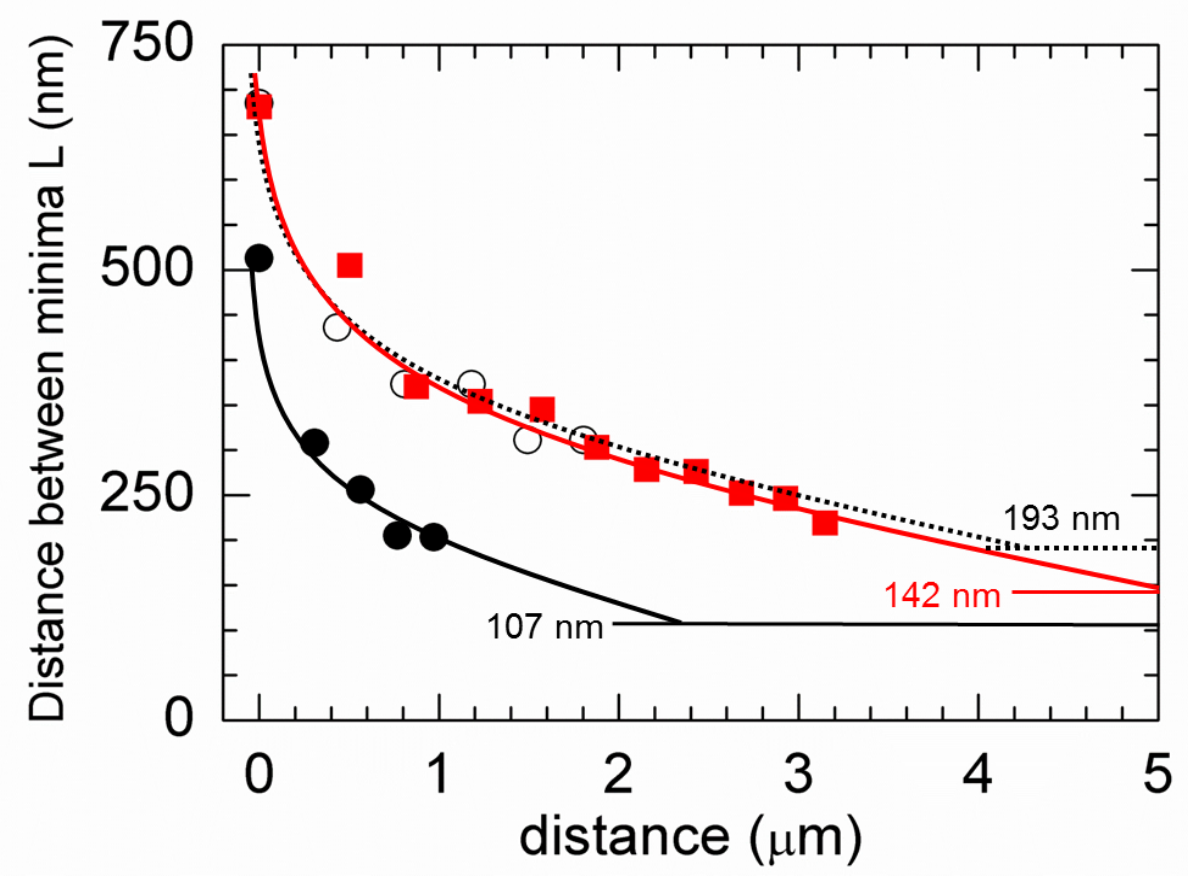

Figure 6. Dependence of the distance between height minima in ONF patterns generated in PTT films with the distance to the position of the first minimum. The pattern was generated by a silica microsphere (diameter $\Phi=4.6 \mu \mathrm{m}$ ) after irradiating with: (a) a single excimer laser pulse irradiation at an angle $\theta=54^{\circ}(\bullet)$, (b) a single excimer laser pulse irradiation at normal incidence (o) and (c) a single Nd:YAG laser pulse irradiation at an angle $\theta=60^{\circ}(\square)$. Lines in the graph are only guides for the eye, including the predicted value of the period in the far field for the different experiments.

Irradiation at normal incidence $\left(\theta=0^{\circ}\right)$ of the PTT film with the same excimer laser produces a pattern of concentric dark rings (Figure 7). In this case, the expected far-field period should be equal to the wavelength of the excimer laser: $p_{b w, c a l c}=\lambda=193 \mathrm{~nm}$ (see Fig. 1). The region around the main ablation crater is less affected by the irradiation (Figure 7, cross section). The period of the structures becomes smaller as the far field is approached (Figure 6, empty black circles), with slightly higher values than in the experiments with $\theta=54^{\circ}$ due to the normal incidence. 
Concerning the optimum fluence range for patterning, we have used fluences in the range of $290-310 \mathrm{~mJ} / \mathrm{cm}^{2}$ (at normal incidence) and $320-400 \mathrm{~mJ} / \mathrm{cm}^{2}$ (at angled incidence). In that range the patterns are well-formed with optimum modulation depth concerning topography and little effect of the laser fluence on the patterning. However, for fluences below this range no ablation occurs at the intensity minima of the patterns, strongly reducing topography modulation and pattern definition. In the opposite case, for too high fluences, the polymer film is completely ablated.

In order to evaluate possible radiation damage effects, Raman spectra for non-irradiated and irradiated PTT films were recorded. Figure 8 compares the Raman spectra of a nonirradiated PTT film with that of a sample irradiated at normal incidence with a single pulse of fluence $300 \mathrm{~mJ} / \mathrm{cm}^{2}$. Since the bands present in both spectra match accurately, it is possible to say that under these irradiation conditions PTT presents chemical stability. Moreover, when zooming in the characteristic region of the $\mathrm{C}=\mathrm{O}$ bond stretching $\left(1720 \mathrm{~cm}^{-1}\right.$, inset in Figure 8$)$, there is no evidence of broadening of this band, which would indicate oxidation effects in the polymer chains. 


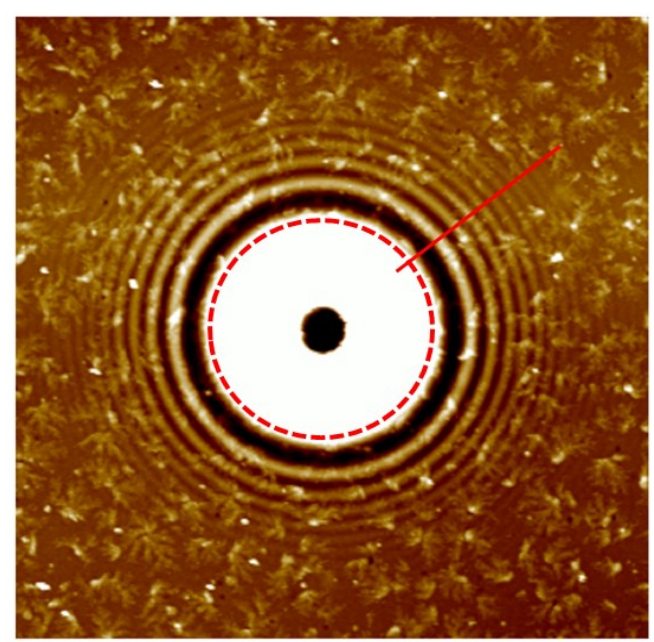

\section{$180 \mathrm{~nm}$}
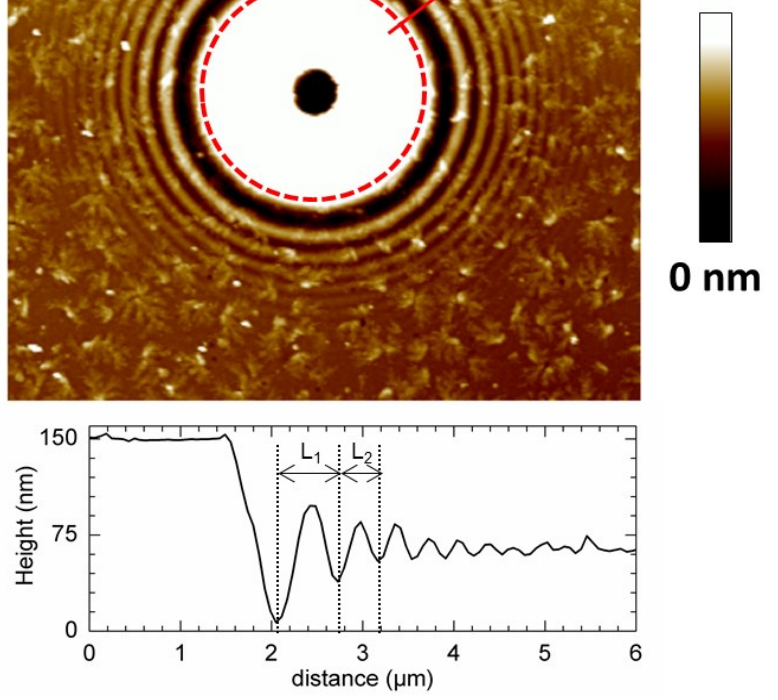

Figure 7. AFM topography map $\left(15 \times 15 \mu \mathrm{m}^{2}\right)$ of the near-field pattern generated on a PTT film by a silica microsphere (diameter $\Phi=4.6 \mu \mathrm{m}$ ), after a single excimer laser pulse irradiation at normal incidence $\left(\theta=0^{\circ}\right)$ and fluence $300 \mathrm{~mJ} / \mathrm{cm}^{2}$. Below, height profile along the depicted red line. The original position of the silica microsphere is marked by a dashed circle. 


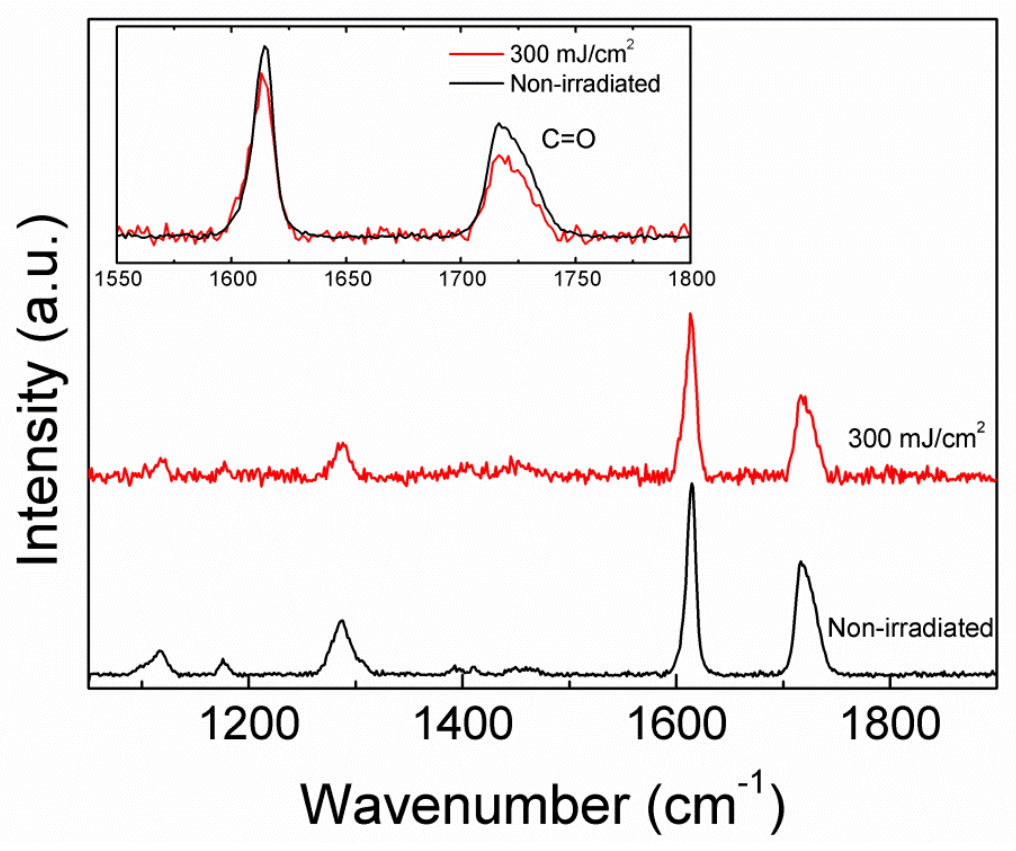

Figure 8. Raman Spectra of non-irradiated and irradiated PTT at normal incidence at a fluence of $300 \mathrm{~mJ} / \mathrm{cm}^{2}$. The inset shows magnification of the region around $1720 \mathrm{~cm}^{-1}$. Fluorescence background has been corrected.

We have also performed patterning experiments using a Nd:YAG laser, in order to investigate the influence of the nature of the laser in the patterning process. Using an angle of incidence of $60^{\circ}$, an elliptical periodic pattern is also observed (Fig. 9a). Unfortunately, a reliable estimation of the local laser fluence was not possible due to the non-homogenous intensity distribution. Thus in the polymer film there are regions where the fluence is higher, leading to stronger ablation (upper left corner in Fig. 9a), than in other regions, where the fluence is appropriate to obtain the desired near-field patterns. The large angle chosen leads to a lengthening of the period in forward direction and shortening in backward direction. Strikingly, one can observe a larger pattern extension with more defined rings (Figure $9 \mathrm{~b}$ and Figure 6). The reason is the much larger coherence length of the Nd:YAG laser which, due to injection seeding, 
extends over the whole pulse length of $3 \mathrm{~m}$, compared to the one of the excimer laser, which is only tens of micrometers. Considering the incidence at $\theta=54^{\circ}$ and the wavelength of the Nd:YAG laser $(266 \mathrm{~nm})$, the asymptotical value of the period in the far field should be $p_{b w, \text { calc }}=\lambda /(1+\sin \theta)=142 \mathrm{~nm}$ (see Figs.1 and 6). Most importantly, no dendritic structures are formed in this case, as opposed to the excimer-produced patterns. The smaller hole is caused by exposure to a second pulse at normal incidence and low fluence $F=2.7 \mathrm{~mJ} / \mathrm{cm}^{2}$, in order to remove the sphere to provide access to the entire patterned region. 

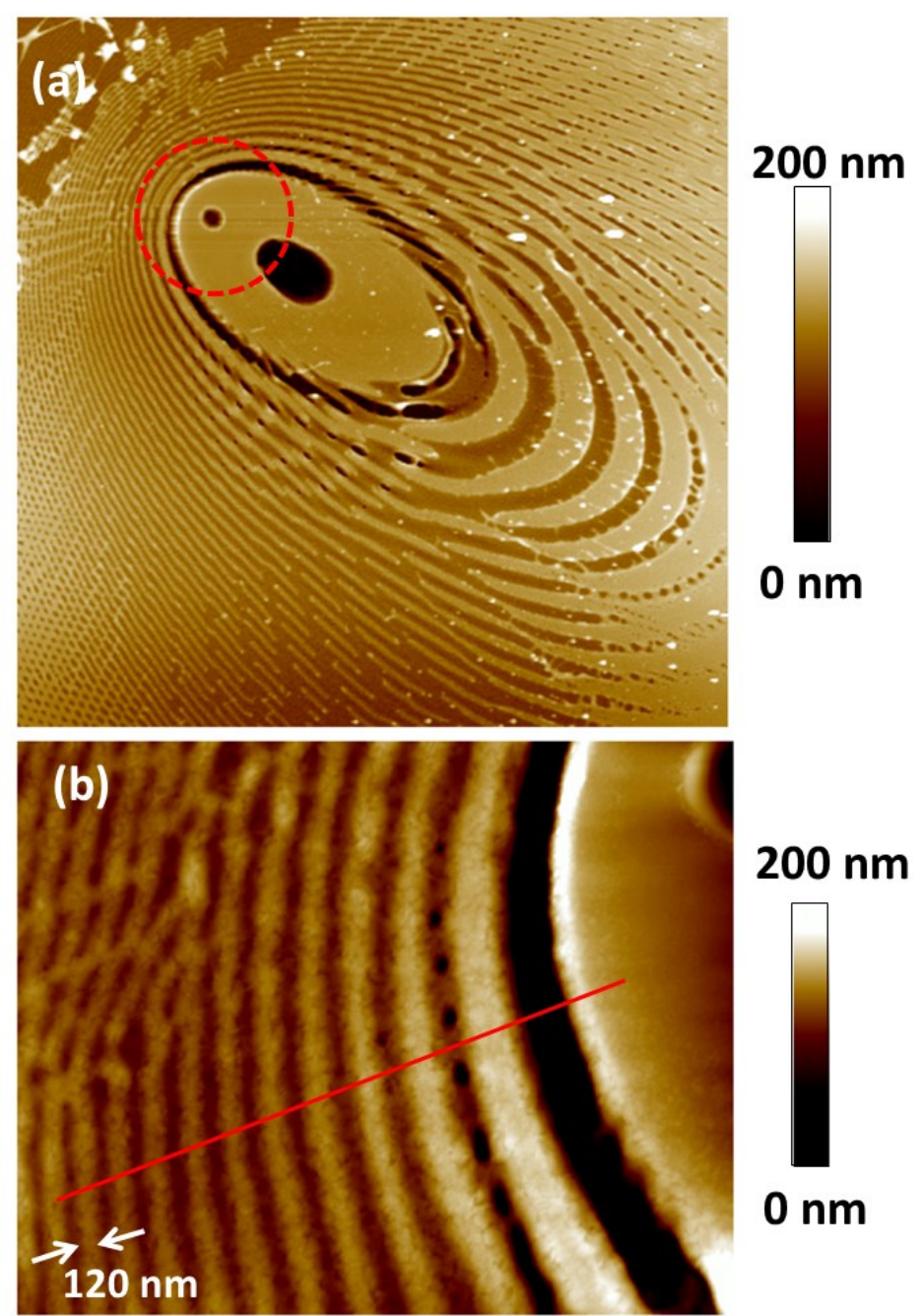

\section{$200 \mathrm{~nm}$}
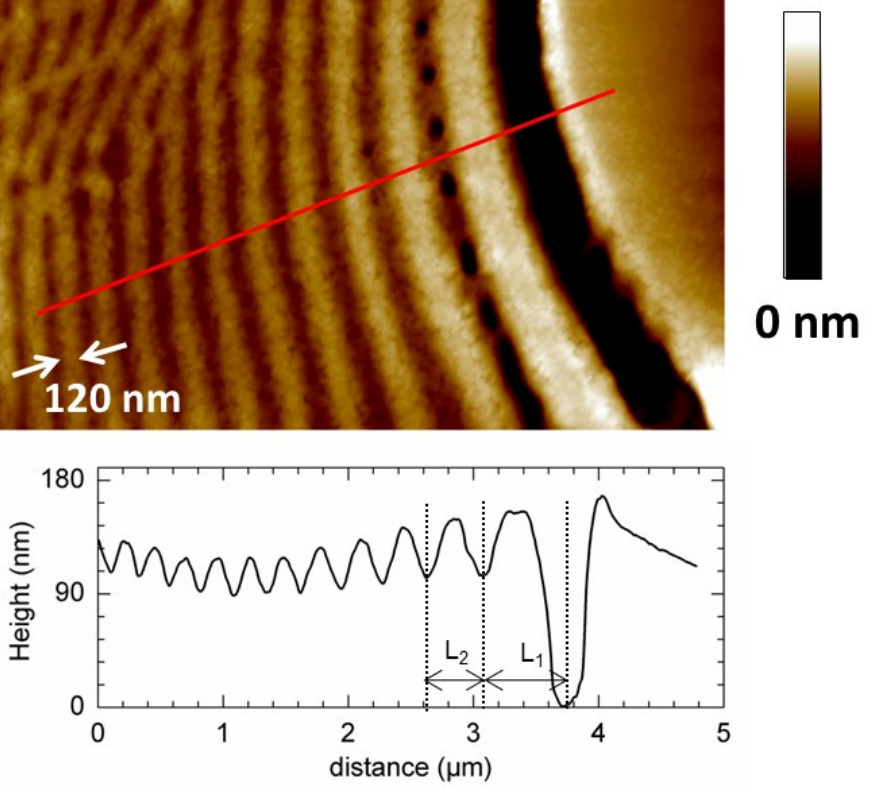

Figure 9. Near-field pattern in PTT film generated by a silica microsphere (diameter $\Phi=4.6 \mu \mathrm{m}$ ) after a single Nd:YAG laser pulse irradiation at an angle $\theta=60^{\circ}$. (a) AFM topography map $\left(25 \times 25 \mu \mathrm{m}^{2}\right)$ and (b) zoom $\left(5 \times 4.5 \mu \mathrm{m}^{2}\right)$ and height profile (below) along the depicted red line. The original position of the silica microsphere is marked by a dashed circle. 
The comparison between the experiments performed with the excimer and the Nd:YAG lasers shows that similar patterns are obtained for both, although some differences associated with the specific properties of each laser can be observed. First, the larger coherence length of the Nd:YAG laser allows to produce more extended patterns than those obtained using the excimer laser. Second, dendritic structures are present on the irradiated PTT films only in the experiments with the excimer laser. At the wavelength of the excimer laser used the linear absorption coefficient of PTT is $\alpha_{193}=186490 \mathrm{~cm}^{-1}$, which, according to Lambert-Beer law, corresponds to an optical penetration depth of $1 / \alpha_{193}=53 \mathrm{~nm}$. Thus, during irradiation an approximately $50 \mathrm{~nm}$ thick layer is strongly heated up, far above the melting temperature of PTT, considering the high fluence values used. ${ }^{33}$ After the laser pulse, the molten layer cools down. During this cooling process PTT may crystallize. ${ }^{34}$ The presence of these dendritic structures on excimerirradiated polymers has been already reported in PET, ${ }^{35}$ and is in agreement with surface crystallization studies in polymer monolayers. ${ }^{36,37}$ The existence of this morphology has been theoretically explained by the fact that in nonequilibrium polymer crystals there is a competition between ordering of individual chains in the crystalline phase and accommodation of incoming comparatively disordered chains at the growth front. $^{38}$ Within this view, after melting of the superficial layer due to the laser irradiation, rearrangement of the polymer chains may occur, leading to the formation of dendritic crystalline structures upon solidification such as the ones present in the PTT films after irradiation (Figure 3a).

In contrast, irradiation with a Nd:YAG laser $\left(\alpha_{266}=25997 \mathrm{~cm}^{-1}\right.$, optical penetration depth $1 / \alpha_{266}=385 \mathrm{~nm}$ ) heats up and melts the whole thickness of the PTT film and the brief time before melt solidification is not sufficient for polymer chains to rearrange and crystallize in a film $150 \mathrm{~nm}$ thick. We attribute the shorter cooling time with the 
$\mathrm{Nd}$ :YAG laser to the fact that the molten layer is much thicker and thus in contact with the Si substrate, acting as an efficient heat sink. Additionally the pulse length of the Nd:YAG, which is a factor of two smaller, contributes to a faster cooling as well.

In order to address the different mechanisms for ONF generated contrast, ONF imprint experiments were performed on an inorganic system, GST, to be compared with the ones carried out in the organic PTT system. The laser was incident at an angle of $\theta=54^{\circ}$ and at a fluence $F=75 \mathrm{~mJ} / \mathrm{cm}^{2}$, generating an elliptical periodic ring pattern with a central elliptical region similar to the ones obtained in PTT, as revealed by optical microscopy (Figure 10). The smaller hole is caused by exposure to a second pulse at normal incidence and low fluence $F=2.7 \mathrm{~mJ} / \mathrm{cm}^{2}$, in order to remove the sphere to provide access to the entire patterned region. Dark rings correspond to regions were the laser fluence has been enhanced locally by the scattered light field of the microsphere. In these regions the local fluence was high enough to trigger amorphization, i.e. the film has been melted and resolidified sufficiently fast to prevent recrystallization. ${ }^{6}$ On the other hand, bright rings and the central ellipse correspond to regions that remain essentially crystalline due to the exposure of a reduced local fluence with respect to the incident fluence. This ring structure becomes clearer by plotting the cross section of the reflectivity along the center of the image (Figure 10 below). The period along the forward direction in the far field has been measured to be $p_{f w, e x p} \approx 1030 \mathrm{~nm}$. This value is in agreement with the expected behaviour, considering incidence at $\theta=54^{\circ}$ and the wavelength of the excimer laser $(\lambda=193 \mathrm{~nm}): p_{f w, c a l c}=\lambda /(1-\sin \theta)=1010 \mathrm{~nm}($ see Fig.1). The maximum optical contrast achieved, excluding the ablation regions, is approximately $20 \%$, which is consistent with this material composition and film thickness. $^{39}$ 

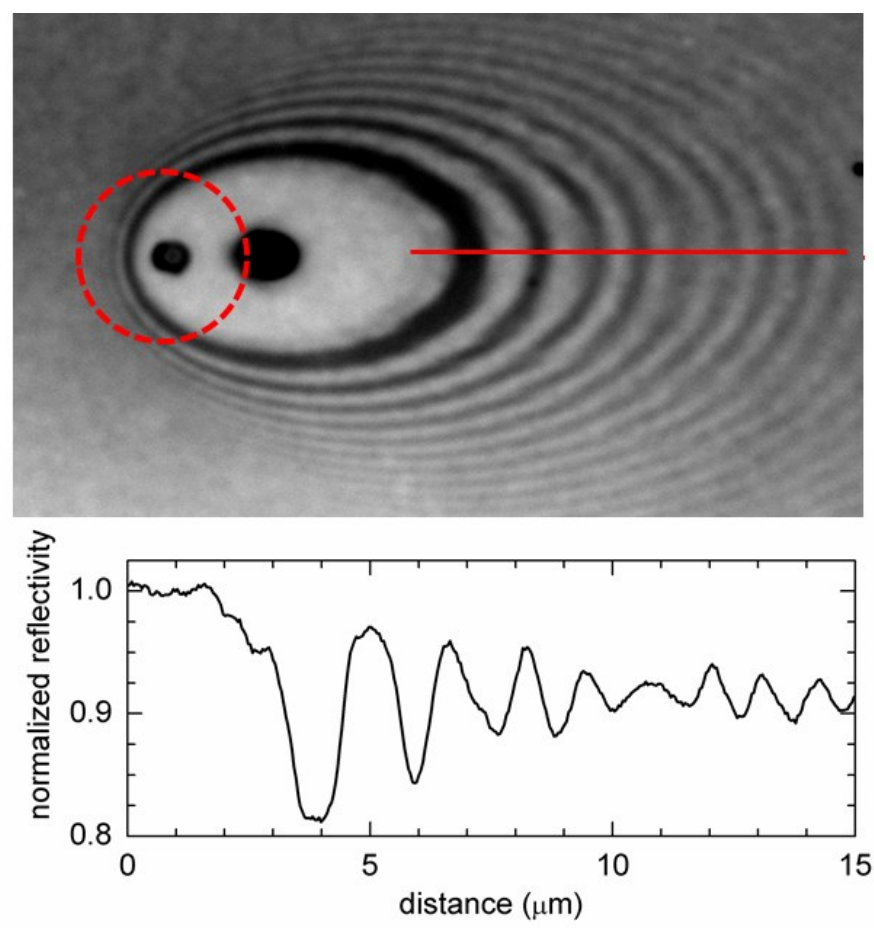

Figure 10. Near-field pattern in GST film generated by a silica microsphere (diameter $\Phi=4.6 \mu \mathrm{m}$ ) after a single excimer laser pulse irradiation at an angle $\theta=54^{\circ}$ (laser impinges from the left) and fluence $75 \mathrm{~mJ} / \mathrm{cm}^{2}$. Optical micrograph $\left(25 \times 15 \mu \mathrm{m}^{2}\right)$ and reflectivity profile (below) along the red line depicted in the image. The original position of the silica microsphere is marked by a dashed circle.

AFM measurements reveal that the imprinted pattern does not only feature optical but also topographic contrast (Figure 11), which originates from the different density of both phases, the amorphous one being lower yielding to an elevation of the amorphous regions over the crystalline film. As can be seen in the topography profile corresponding to the zoomed region (Figure 11b), the maximum contrast between crystalline and laser-amorphized regions is $6.5 \mathrm{~nm}$. This value is higher than reported in ${ }^{40}$, indicating that additional processes have taken place. It is worth noting that there is a significant difference in roughness between amorphous and crystalline regions, amorphous being smoother. 

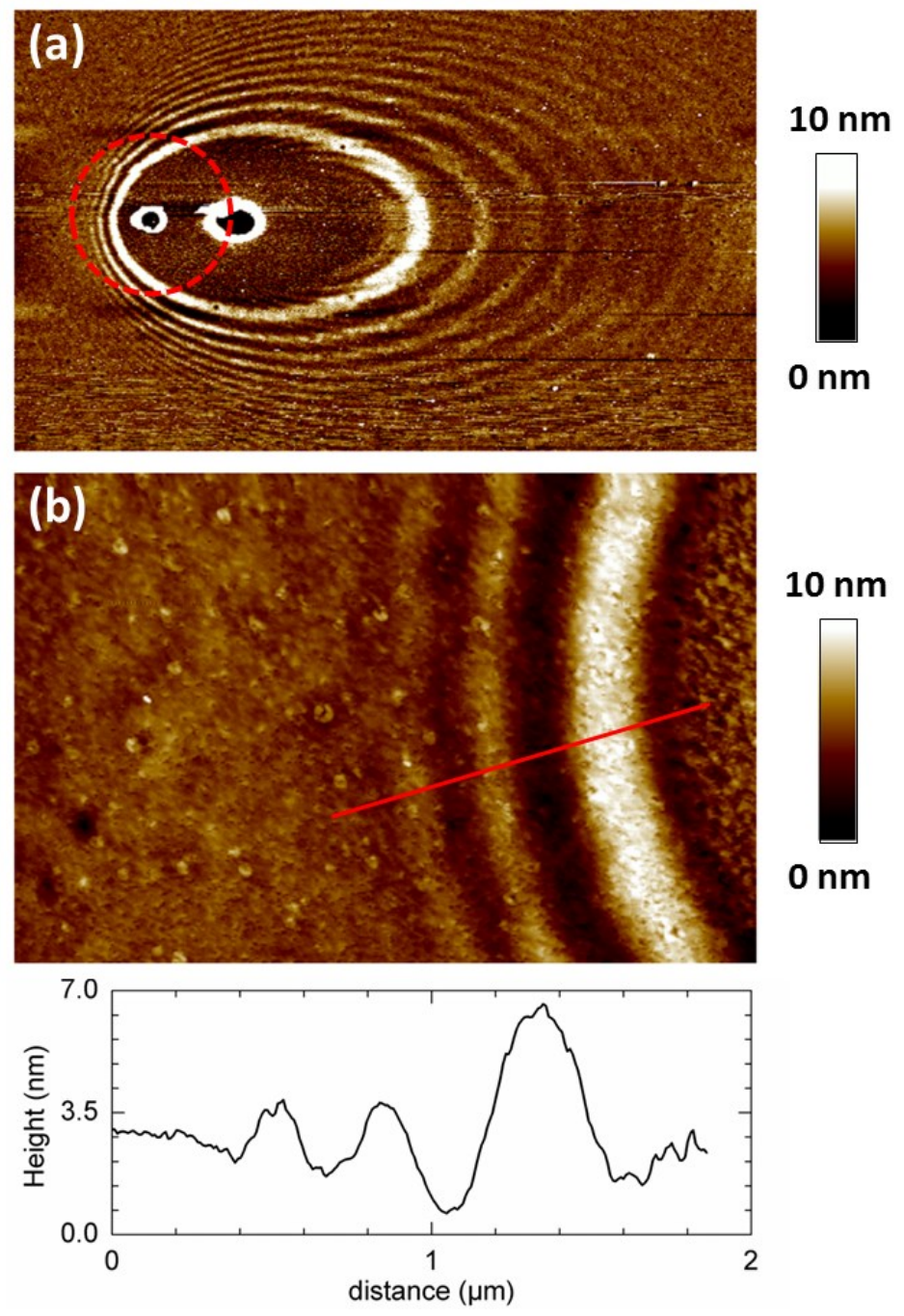

Figure 11. Near-field pattern in GST film generated by a silica microsphere (diameter $\Phi=4.6 \mu \mathrm{m}$ ) after a single excimer laser pulse irradiation at an angle $\theta=54^{\circ}$ (laser impinges from the left) and fluence $75 \mathrm{~mJ} / \mathrm{cm}^{2}$. (a) AFM topography map $\left(25 \times 15 \mu \mathrm{m}^{2}\right)$ and (b) zoom $\left(3 \times 2 \mu \mathrm{m}^{2}\right)$ and topography profile (below) along the red line in the backscattering region. The original position of the silica microsphere is marked by a dashed circle.

\section{CONCLUSIONS}

Summing up, we have achieved to imprint and characterize the optical near field of silica microspheres on PTT and GST thin films upon irradiation with single laser pulses. 
In contrast to the phase change mediated patterning process in GST, the responsible mechanism in PTT films is near field ablation. The form of the pattern consists of elliptical or circular periodic rings, depending on the angle of incidence of the laser, and features a complex substructure on the nanoscale. In the polymer the topography modulation ranges over the entire film thickness and minimum feature sizes as small as $120 \mathrm{~nm}$ have been imprinted. The specific properties of the laser employed have a strong impact on the characteristics of the pattern, in particular the laser wavelength and coherence length. The optical penetration depth of the laser light in the material has been identified as an important factor for suppressing the formation of dendritic structures. The experiments presented in this work extend the application of optical near-field distributions to nanopatterning of polymeric films in a single step process.

\section{AUTHOR INFORMATION}

\section{Corresponding Author}

* E-mail: i.fabiani@csic.es

\section{Notes}

The authors declare no competing financial interest.

\section{ACKNOWLEDGMENT}

The authors acknowledge financial support from the MINECO (grants MAT201123455, MAT2012-33517, TEC2011-22422 and FPI BES-2010-030074). We thank A. Szymczyk and Z. Rosłaniec for providing PTT samples and E. Rebollar for the absorbance measurements of PTT thin films.

\section{REFERENCES}

(1) Zheludev, N. I. Nat. Mater. 2008, 7, 420-422. 
(2) Durig, U.; Pohl, D. W.; Rohner, F. J. Appl. Phys. 1986, 59, 3318-3327.

(3) McLeod, E.; Arnold, C. B. Nat. Nanotechnol. 2008, 3, 413-417.

(4) Valev, V. K.; De Clercq, B.; Biris, C. G.; Zheng, X.; Vandendriessche, S.; Hojeij, M.; Denkova, D.; Jeyaram, Y.; Panoiu, N. C.; Ekinci, Y.; Silhanek, A. V.; Volskiy, V.; Vandenbosch, G. A. E.; Ameloot, M.; Moshchalkov, V. V.; Verbiest, T. Adv. Mater. 2012, 24, OP208-OP215.

(5) Leiprecht, P.; Kuehler, P.; Longo, M.; Leiderer, P.; Afonso, C. N.; Siegel, J. Appl. Phys. Lett. 2011, 98.

(6) Kuehler, P.; Garcia de Abajo, F. J.; Solis, J.; Mosbacher, M.; Leiderer, P.; Afonso, C. N.; Siegel, J. Small 2009, 5, 1825-1829.

(7) Lippert, T. Laser application of polymers. In Polymers and Light; Lippert, T., Ed.; Springer: Berlin, 2004; pp 51-246.

(8) Lasagni, A.; Holzapfel, C.; Weirich, T.; Mucklich, R. Appl. Surf. Sci. 2007, 253, 8070-8074.

(9) Wolferen, H. V.; Abelmann, L. Laser Interference Lithography. In Lithography: Principles, Processes and Materials; Hennessy, T. C., Ed.; Nova Science Publishers, Inc.: New York, 2011; pp 133-148.

(10) Plech, A.; Kotaidis, V.; Lorenc, M.; Boneberg, J. Nat. Phys. 2006, 2, 44-47.

(11) Geldhauser, T.; Kolloch, A.; Murazawa, N.; Ueno, K.; Boneberg, J.; Leiderer, P.; Scheer, E.; Misawa, H. Langmuir 2012, 28, 9041-9046.

(12) Kramer, A.; Trabesinger, W.; Hecht, B.; Wild, U. P. Appl. Phys. Lett. 2002, 80, $1652-1654$.

(13) Brodoceanu, D.; Landstrom, L.; Bauerle, D. Appl. Phys. A: Mater. Sci. Process. 2007, 86, 313-314. 
(14) Morarescu, R.; Englert, L.; Kolaric, B.; Damman, P.; Vallee, R. A. L.; Baumert, T.; Hubenthal, F.; Traeger, F. J. Mater. Chem. 2011, 21, 4076-4081.

(15) Wang, Z. B.; Hong, M. H.; Luk'yanchuk, B. S.; Lin, Y.; Wang, Q. F.; Chong, T. C. J. Appl. Phys. 2004, 96, 6845-6850.

(16) Kuehler, P.; Javier Garcia de Abajo, F.; Leiprecht, P.; Kolloch, A.; Solis, J.;

Leiderer, P.; Siegel, J. Opt. Express 2012, 20, 22063-22078.

(17) Chen, D.; Zhao, W.; Russell, T. P. Acs Nano 2012, 6, 1479-1485.

(18) Rebollar, E.; Sanz, M.; Perez, S.; Hernandez, M.; Martin-Fabiani, I.; Rueda, D. R.; Ezquerra, T. A.; Domingo, C.; Castillejo, M. Phys. Chem. Chem. Phys. 2012, 14, 15699-15705.

(19) Schift, H. J. Vac. Sci. Technol. B 2008, 26, 458-480.

(20) Xia, L.; Wei, Z. X.; Wan, M. X. J. Colloid Interface Sci. 2010, 341, 1-11.

(21) Hu, Z. J.; Tian, M. W.; Nysten, B.; Jonas, A. M. Nat. Mater. 2009, 8, 62-67.

(22) Pyda, M.; Boller, A.; Grebowicz, J.; Chuah, H.; Lebedev, B. V.; Wunderlich, B. J. Polym. Sci. Pt. B-Polym. Phys. 1998, 36, 2499-2511.

(23) Lewin, M. In Handbook of Fiber Chemistry, 3rd ed.; CRC Press: Boca Raton, 2007.

(24) Wang, Y. Q.; Zhu, H.; Li, B. J. Opt. Commun. 2011, 284, 3276-3279.

(25) Yu, H.; Liao, D.; Johnston, M. B.; Li, B. Acs Nano 2011, 5, 2020-2025.

(26) Xing, X.; Zhu, H.; Wang, Y.; Li, B. Nano Lett. 2008, 8, 2839-2843.

(27) Martin-Fabiani, I.; Rebollar, E.; Perez, S.; Rueda, D. R.; Garcia-Gutierrez, M. C.; Szymczyk, A.; Roslaniec, Z.; Castillejo, M.; Ezquerra, T. A. Langmuir 2012, 28, 79387945. 
(28) Rueda, D. R.; Martin-Fabiani, I.; Soccio, M.; Alayo, N.; Perez-Murano, F.;

Rebollar, E.; Garcia-Gutierrez, M. C.; Castillejo, M.; Ezquerra, T. A. J. Appl.

Crystallogr. 2012, 45, 1038-1045.

(29) Mie, G. Ann. Phys.-Berlin 1908, 25, 377-445.

(30) Leiderer, P.; Bartels, C.; Konig-Birk, J.; Mosbacher, M.; Boneberg, J. Appl. Phys. Lett. 2004, 85, 5370-5372.

(31) Luo, W.-a.; Liao, Z.; Yan, J.; Li, Y.; Chen, X.; Mai, K.; Zhang, M.

Macromolecules 2008, 41, 7513-7518.

(32) Chuang, W.-T.; Su, W.-B.; Jeng, U. S.; Hong, P.-D.; Su, C.-J.; Su, C.-H.; Huang, Y.-C.; Laio, K.-F.; Su, A.-C. Macromolecules 2011, 44, 1140-1148.

(33) Rebollar, E.; Perez, S.; Hernandez, J. J.; Martin-Fabiani, I.; Rueda, D. R.;

Ezquerra, T. A.; Castillejo, M. Langmuir 2011, 27, 5596-5606.

(34) Xue, M. L.; Sheng, J.; Yu, Y. L.; Chuah, H. H. Eur. Polym. J. 2004, 40, 811-818.

(35) Heitz, J.; Arenholz, E.; Bauerle, D.; Hibst, H.; Hagemeyer, A.; Cox, G. Appl. Phys. A: Mater. Sci. Process. 1993, 56, 329-333.

(36) Reiter, G.; Sommer, J. U. J. Chem. Phys. 2000, 112, 4376-4383.

(37) Ma, Z.; Zhang, G.; Zhai, X.; Jin, L.; Tang, X.; Yang, M.; Zheng, P.; Wang, W. Polymer 2008, 49, 1629-1634.

(38) Sommer, J. U.; Reiter, G. J. Chem. Phys. 2000, 112, 4384-4393.

(39) Siegel, J.; Gawelda, W.; Puerto, D.; Dorronsoro, C.; Solis, J.; Afonso, C. N.; de Sande, J. C. G.; Bez, R.; Pirovano, A.; Wiemer, C. J. Appl. Phys. 2008, 023516.1-7. (40) Weidenhof, V.; Friedrich, I.; Ziegler, S.; Wuttig, M. J. Appl. Phys. 1999, 86, 58795887. 